\title{
Emotional Evaluation Based on SVM
}

\author{
Yu Nie ${ }^{1,}$, Yang Wu, Zhongyao Yang, Guangzhi Sun, Yongjian Yang and \\ Xuanyi Hong \\ ${ }^{1}$ College of Software, Jilin University, Jilin, China \\ a13943186112@163.com
}

Keywords:Data Mining, SVM, Bioinformatics, EEG, Machine Learning.

\begin{abstract}
With the continuous development of the modern intelligent household, people found that brain wave can be used for controlling household appliances. EEG signal, as a typical brain wave signal carrying the brain state information, has walked into the researcher's view. Brain wave carries detailed information about the state of the brain. As a result, using computer to collect and analyze EEG signal plays a great role in smart home. This paper uses positive and negative emotional EEG signal as the research object, begins with introducing the research status of brain waves, and then uses the Chinese Affective Picture System (CAPS [12]) of Chinese Academy of Sciences, designs the watch pictures to experiment, utilizes machine learning algorithm of support vector machine (SVM) for data analysis, and obtains an accuracy of 58.3\% eventually. This paper provides a feasible scheme for the study of EEG in the field of emotion analysis.
\end{abstract}

\section{Introduction}

In 1884, the father of American psychology James put forward that emotion was a kind of feeling of people for their body changes, the change of the body appeared before emotion perception and any emotion produced must be accompanied by some changes of the body[1]. This is the earliest definition of emotion. In 1927, Connon thought the mood was determined by the hypothalamus[2]. In 1937, Papez linked emotions to physical activity[3]. At present, a large number of results of neurological and cognitive science research show that the generation of emotion is related to physiological activities, particularly the activity of the brain which provides a theoretical basis for analyzing the activities of the cerebral cortex for emotion recognition.

Emotional classification, like emotion, has not yet formed a unified theory. Modern scholars through research and experiment put forward their own emotional set, such as James's emotional set, Ekman's emotional set, etc. Through further research, the researchers found that there is a certain correlation between emotions, such as anger and disgust sometimes both appear, so there is a two-dimensional Lange emotional classification model[4]. The model is also the most common classification model using the longitudinal expression of mood pleasure, dislike to gradually over like; use abscissa to indicate the state of excitement, from depression to excitement.

Common emotion recognition methods have two categories. The first one is based on the recognition of non-physiological signals, including the recognition of facial expressions and the recognition of speech tones. The advantage is that data acquisition is relatively simplewithoutthe need of special equipment. The disadvantage is that the reliability of emotion recognition is not ensured, because the data can be subject to subjective factors. The second one is based on physiological signal recognition.At present, the main physiological signals have EOG, GSP,BVP,EMG,EEG, ECG,etc.

This paper uses the brain waves in physiological signals for emotion recognition, through the acquisition, identification, analysis of EEG signals. The main steps of emotion recognition based on EEG include: emotional evoked, EEG signal collection, EEG preprocessing, feature extraction, feature dimension reduction, emotion pattern learning and classification.First, we design the relevant experiments for emotional evoked. Second, we normalize the data extracted from EEG signals. Finally we use support vector machine model for data training and testing, to get the classification results. 
Research on emotion recognition is of great significance.An indicator of artificial intelligence is to understand human emotions, so emotional recognition related research will promote the development of artificial intelligence.For human-computer interaction, let the machine understand human emotions, will make the understanding of human intentions more accurate to help people better.There are many applications of emotion recognition, such as driver fatigue detection, heart patient care, etc.

At present, there are many researches on emotion recognition based on EEG signal[9,10,11].At the stage of learning and classifying emotions, the algorithm often used is support vector machine.In this paper, support vector machine is usedfor recognizing positive and negative emotions.

\section{Data Collection}

\section{Data Source}

The first step of emotional classification is to collect original brain wave data. Some experiments must be taken to stimulate the experimenters act as expect. That's the proper way to collect the real brain wave data.

This study uses the Osgood's emotional theory. Namely, the three main dimensions of emotion are valence, arousal and dominance. The researchers select a lot of positive and negative pictures from Chinese Affective Picture System(CAPS[12]) according to this theory.

\section{Equipment}

In this research, we choose MindBind Research, developed by Neurosky, for EEG data collection. MindBind Research consists of two electrodes whose distribution refers to the international 10-20 system [5] (shown in Fig.1), with a sampling frequency of $512 \mathrm{~Hz}$.
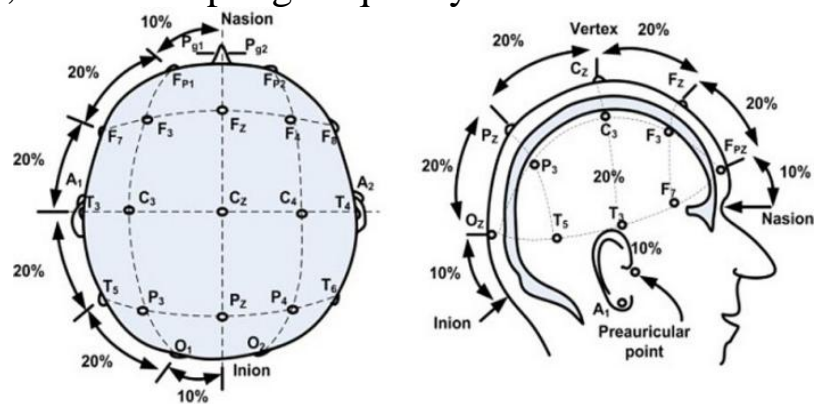

Fig.1. International 10-20 system

Data is transmitted via a Standard Parallel Port [6]. Using Bluetooth dongle with corresponding driver, we are able to connect MindBind with computer.

In order to implement and maintain stable data transmission, we write a Win32 Console Application, utilizing provided API and communication driver. This application acquires the version of current communication driver, then it checks whether the equipment has been connected to computer or not. If true, it starts receiving data and outputtinginformation about whether data had been received through a Win32 console.

\section{Experimental Procedure}

The experiment was carried out in a seminar room, subjects were asked to seat 2 meters away from the screen where fixed size pictures were displayed. Positive and negative type pictures were displayed randomly so that the appearance of each picture is unpredictable. In order to smooth the emotion of subjects, a piece of soothing music was played before the experiment started. Five pictures were also displayed as a pilot set after the music to ensure subjects were familiar with experimental process[7]. 
$2 \mathrm{~s}$
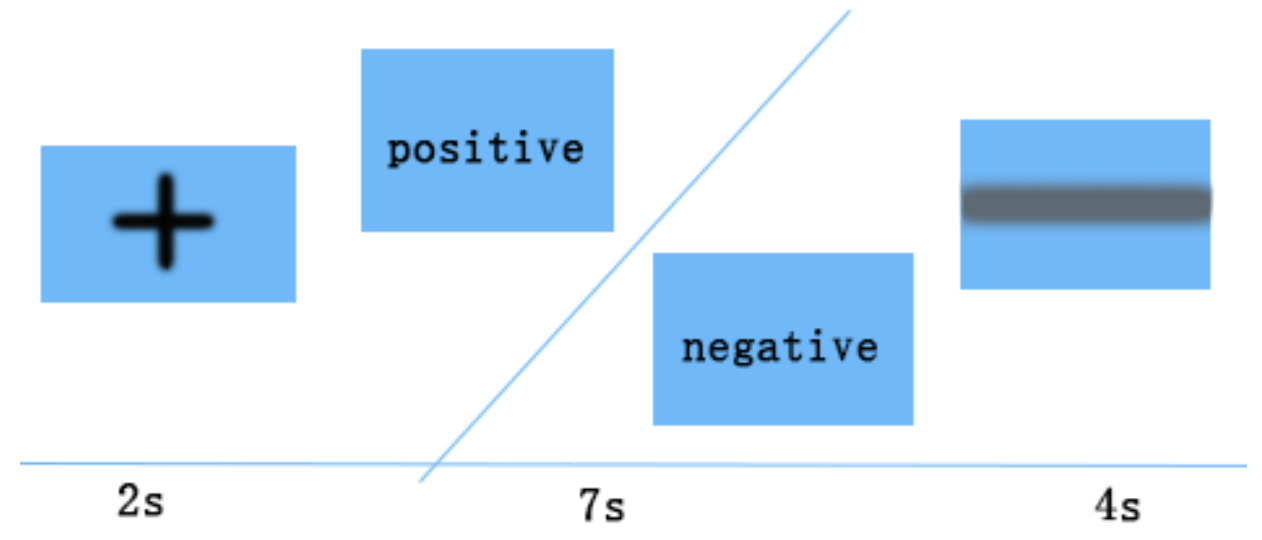

Fig. 2. Sample trial

The experiment consists of several trials. As shown in Fig.2, each trial begins with ablack fixation cross in the center of the screen which lasted for $2000 \mathrm{~ms}$. Then a picture is displayed and lasts for $7000 \mathrm{~ms}$. After that, a gray region appears and lasts for $4000 \mathrm{~ms}$. Such process is repeated 30 times. Meanwhile, researchers record the sequence of pictures and noticeable reactions of subjects, such as facial expressions and body movements.

\section{Experiments and Results}

Normalization

Data could be mapped into interval $[0,1]$ by normalization, which eliminates the effects of units and turns original data into dimensionless values. In this research, we used standard score normalization, which is also known as z-score normalization [8]. Z-score was computed by

$$
z=\frac{x-\mu}{\delta}
$$

In this experiment, normalization was implemented by subtracting the average of each column from each value of this column and then dividing the results by the standard deviation of this column. Normalized data is subject to standard normal distribution, with a mean of 0 and a standard deviation of 1 .

Training and Classification

Experiment was carried out on the test data using the algorithm of SVM classification.

After normalization, data need to be tagged for supervised learning. In this research, only positive and negative emotions were taken into consideration. We tagged each data according to the order of pictures recorded by researchers and the duration of each picture.The average accuracy of the classification of positive and negative emotions on the test set was $58.3 \%$. The distribution of data is shown in Fig. 3.
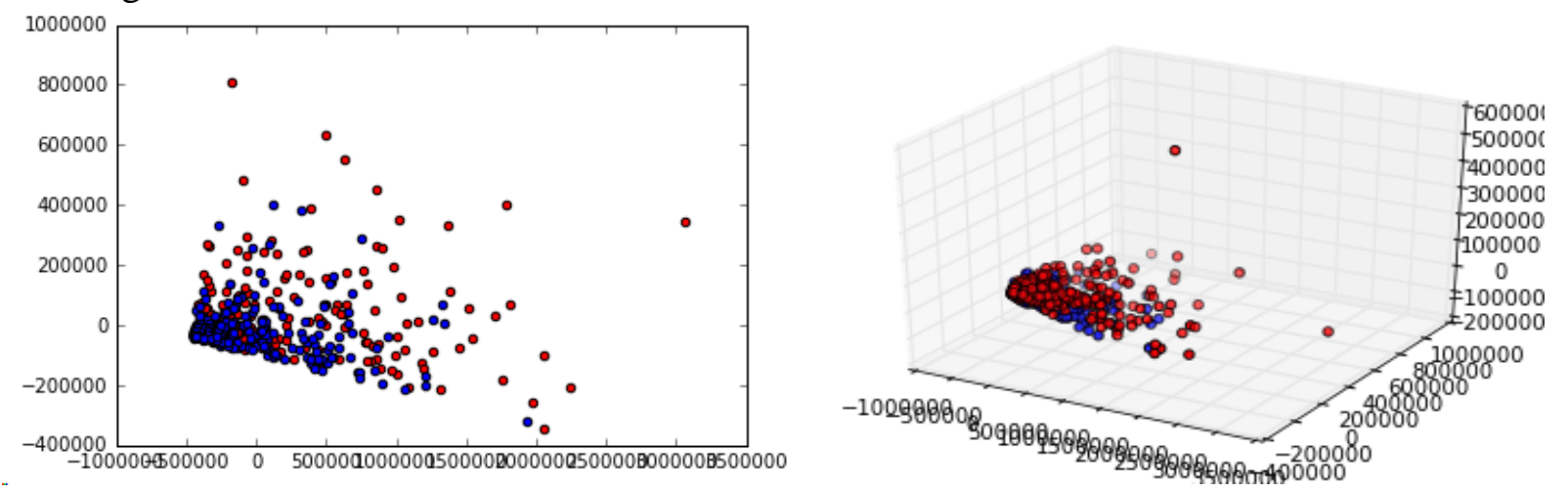

Fig. 3. The distribution of data 
An accuracy of $58.3 \%$ is not ideal, as a result, the researchers made reflection and conclusion on the experimental process. The main factor that may affect the result might be that the projection equipment, the EEG-collection equipment and the data analysis equipment were unsynchronized, which led to a time difference within one second. However, the data was tagged once per second, therefore such time difference could cause an error in the result.

\section{Conclusion and Future Work}

\section{Conclusions}

In this research, a large amount of EEG raw data was collected from hundreds of college students, which was separated by the use of SVM. Consequently, emotion recognition was realized. This study got a conclusion: the EEG data is non-linear separable, and therefore the existing Bayesian classification algorithm is not effective for EEG classification.

Future Work

In view of most of the pictures in the CAPS [12] were updated years ago, the results of the experiments are affected to varying degrees. We hope this problem can be solved sooner or later and the result of emotional classification can be better in the future.

\section{References}

[1] W. James. Mind 34 (1884): 188-205.

[2] W.B. Cannon. The American journal of psychology 39.1/4 (1927): 106-124.

[3] T. Dalgleish. Nature Reviews Neuroscience 5.7 (2004): 583-589.

[4] P.J. Lang, M.M. Bradleyand B.N. Cuthbert. "International affective picture system (IAPS): Technical manual and affective ratings." Gainesville, FL: The Center for Research in Psychophysiology, University of Florida (1999).

[5] H.H. Jasper. Electroencephalography and clinical neurophysiology 10 (1958): 371-375.

[6] C. Peacock. "Interfacing the standard parallel port." (1998).

[7] K. Schaaffand T. Schultz. "Towards emotion recognition from electroencephalographic signals." 2009 3rd International Conference on Affective Computing and Intelligent Interaction and Workshops. IEEE, 2009.

[8] A. Jain, K.Nandakumarand A. Ross. Pattern recognition 38.12 (2005): 2270-2285.

[9] B. Kahn. Electroencephalogram (EEG) signal processing, wave identification, and emotion recognition. Diss. California State University, Northridge, 2015.

[10]R.M. Mehmoodand H.J. Lee. Int. J. Bio-Sci. Bio-Technol. 7 (2015): 23-32.

[11]J. Atkinsonand D. Campos. Expert Systems with Applications 47 (2016): 35-41.

[12]L. Bai, H. Ma, et al. Chinese Mental Health Journal 19.11 (2005): 719-722. 